\title{
A Noncooperative View of Consistent Bankruptcy Rules*
}

\author{
Nir D agan \\ Department of Economics, Universitat Pompeu Fabra, Barcelona, Spain
}

and

R oberto Serrano and O scar V olij

Department of Economics, Brown University, Providence, Rhode Island 02912

R eceived February 7, 1995

\begin{abstract}
We introduce a game form that captures a noncooperative dimension of the consistency property of bankruptcy rules. A ny consistent and monotone rule is fully characterized by a bilateral principle and consistency. Like the consistency axiom, our game form, together with a bilateral principle, yields the corresponding consistent bankruptcy rule as a result of a unique outcome of $\mathrm{N}$ ash equilibria. The result holds for a large class of consistent and monotone rules, including the Constrained Equal A ward, the Propositional Rule, and many other well known rules. Moreover, all of the subgame perefect equilibria are coalition-proof in the associated game in strategic form. Journal of Economic Literature Classification N umbers: C 72 and D 63. (c) 1997 A cademic Press
\end{abstract}

\section{INTRODUCTION}

The consistency property has proved very powerful in characterizing some of the most important solution concepts in cooperative game theory (see, for example, the characterizations of the core and the pre-kernel by

* A large part of this work was done while Dagan was at the Hebrew U niversity of Jerusalem and Universidad Carlos III de Madrid, and while Volij was at the Hebrew U niversity and CentER of E conomic R esearch at Tilburg. Serrano gratefully acknowledges the hospitality of the Center of Rationality at the Hebrew U niversity of Jerusalem and U niversitat Pompeu Fabra of Barcelona. We are grateful to $M$ ichael M aschler for very useful comments. 
Peleg (1986) and of the $\mathrm{N}$ ash bargaining solution by Lensberg (1988)). ${ }^{1}$ However, consistency alone does not isolate a unique rule in bankruptcy problems, even after restricting attention to symmetric, scale-invariant, and monotone rules. On the other hand, a simple extension of a result of A umann and M aschler's (1985) shows that any monotone and consistent rule is completely characterized by a two-person rule and consistency.

Consistency has also been suggested as a valuable guide in designing noncooperative mechanisms that implement some cooperative solutions (see, for example, Krishna and Serrano, 1996). Namely, extensive forms can be constructed whose subgames relate to the respective reduced cooperative problems. By concentrating on the subgame-perfect equilibria of such mechanisms, one can hope to implement the underlying consistent solution. This paper provides additional support for the idea that consistency is a useful tool in the Nash program for cooperative games.

We introduce a game in extensive form that captures a noncooperative dimension of the consistency property of bankruptcy rules. In the game one of the creditors with the highest claim must make a proposal about how to split the estate. Those creditors who accept the proposal receive their shares, and those who reject may "appeal to the bilateral court" that stands as an outside option. Our game form generates a large family of consistent and monotone bankruptcy rules presented in the axiomatic theory. It takes a two-person rule as an input and yields the unique consistent generalization of that rule as an output. The unique equilibrium outcome of the game associated with a specific two-person rule is the allocation recommended by the unique consistent generalization of that rule. In this sense our game form operates like the consistency property in the axiomatic approach, capturing a noncooperative dimension of consistency in the framework of bankruptcy problems. That is, by replacing the consistency axiom in A umann and M aschler's (1985) result, our game form provides its noncooperative counterpart.

Like other games based on consistency, our game allows for "partial agreements," where a player cannot be prevented from getting his offered share if he is happy with it. The question arises of whether such equilibria are coalitionally stable. Could the proposer offer a larger fraction of the pie to a creditor and then split it with him? When deviations are "coalitionally credible," the answer is negative: although they are not strong Nash, we show that all the subgame-perfect equilibria of the game are coalition-proof.

We will assume throughout that the claims are known by everybody (including the court). As discussed above, our focus is the noncooperative dimension of the consistency axiom in bankruptcy problems. In Dagan et

\footnotetext{
${ }^{1}$ For a good survey, see Thomson (1990) or Thomson (1996b).
} 
al. (1995), we analyze the related problem of implementing bankruptcy rules when the claims are unknown to the court.

The paper is organized as follows: Section 2 is devoted to the axiomatic treatment of bankruptcy problems. Section 3 discusses the relation between bilateral principles of justice and consistency. The multilateral noncooperative model and the main result are presented in Section 4. Coalition-proofness is discussed in Section 5. A result concerning strictly monotone rules is the object of Section 6, and Section 7 concludes.

\section{THE AXIOMATIC BANKRUPTCY MODEL}

A bankruptcy problem is a pair $(E ; d)$ where $d \in \mathbb{R}_{+}^{I}$ is a vector of non-negative real numbers (claims), indexed by some finite non-empty subset $I$ of natural numbers (creditors), and $0 \leq E \leq \sum_{i \in I} d_{i}:=D$. E is the estate to be allocated, and $D$ is the sum of the claims.

A $n$ allocation in $(E ; d)$ is a vector $x \in \mathbb{R}_{+}^{I}$ such that $\sum_{i \in I} x_{i}=E$ and $x_{i} \leq d_{i}$ for all $i \in I$. The set of all allocations in $(E ; d)$ is denoted by $\mathscr{A}(E ; d)$.

Remark. For any list of claims $d \in \mathbb{R}_{+}^{I}$, any vector $x \in \mathbb{R}_{+}^{I}$ with $x_{i} \leq d_{i}$ is an allocation of the bankruptcy problem $\left(\sum_{i \in I} x_{i} ; d\right)$. Therefore, when there is no danger of confusion, we shall call any such vector $x$ an allocation without specifying the bankruptcy problem to which it refers.

A rule is a function that assigns to each bankruptcy problem a unique allocation.

EXAMPLES. (a) The proportional rule,

$$
\operatorname{Pr}(E ; d)=\lambda d
$$

where $\lambda D=E$.

The proportional rule, widely applied nowadays, allocates awards in proportion to claim size. The proportionality principle was favored by the philosophers of ancient $\mathrm{G}$ reece, and A ristotle even considered it as equivalent to justice.

(b) The constrained equal award (CE A) rule:

$$
\operatorname{CEA}(E ; d)=x
$$

where $x_{i}=\min \left(\lambda, d_{i}\right)$ and $\lambda$ solves the equation $\sum_{i \in I} \min \left(\lambda, d_{i}\right)=E^{2}$ This rule assigns the same sum to all creditors as long as it does not exceed

\footnotetext{
${ }^{2}$ This equation has a unique solution when $D>E$. If $D=E$, any solution $\lambda$ is greater than or equal to the maximum claim and therefore $x_{i}=d_{i}$ for all $i$.
} 
each creditor's claim. This rule is also very ancient, and was adopted by important rabbinical legislators, including $M$ aimonides.

(c) The constrained equal loss (CEL) rule:

$$
\operatorname{CEL}(E ; d)=x
$$

where $x_{i}=\max \left(0, d_{i}-\lambda\right)$ and $\lambda$ solves the equation $\sum_{i \in I} \max \left(0, d_{i}-\lambda\right)$ $=E .^{3}$ This rule assigns losses $\left(d_{i}-x_{i}\right)$ in the same manner as the CEA assigns awards.

(d) The Pineles Rule:

$\operatorname{Pin}(E ; d)=\operatorname{CEA}(\min \{D / 2, E\} ; d / 2)+\operatorname{CEA}(\max \{E-D / 2,0\} ; d / 2)$.

When the estate does not exceed half the sum of the claims, the Pineles rule assigns each creditor a fixed amount, as long as it does not exceed half his claim (otherwise, it assigns him half his claim). When the estate exceeds half the sum of the claims, it first gives each creditor half his claim and then divides the remainder (which, by definition, cannot exceed half the sum of the claims) according to the procedure described in the previous sentence. This rule appears in Pineles (1861, p. 64), and is an interpretation of a controversial mishna (Ketuboth 93).

(e) The Contested Garment Consistent (CG C) Rule:

$\operatorname{CGC}(E ; d)=\operatorname{CEA}(\min \{D / 2, E\} ; d / 2)+\operatorname{CEL}(\max \{E-D / 2 ; 0\} ; d / 2)$.

This rule was proposed by A umann and M aschler (1985) as an alternative interpretation of the mishna mentioned above.

(f) Equal Sacrifice Rules: Let $U: \mathbb{R}_{++} \rightarrow \mathbb{R}$ be a continuous and strictly increasing function that satisfies $\lim _{x \rightarrow 0} U(x)=-\infty$. The equal sacrifice rule $\phi$ relative to $U$ satisfies

$$
\begin{array}{r}
\phi(E ; d)=x \Leftrightarrow \exists c \geq 0 \text { such that } \forall i \in I \text { with } d_{i}>0, \\
\\
U\left(d_{i}\right)-U\left(x_{i}\right)=c, \text { when } E>0 .
\end{array}
$$

These rules assign awards so as to equalize absolute sacrifice evaluated according to a prespecified utility function. Note that the equal sacrifice rule with respect to the logarithmic function is the proportional rule. The equal sacrifice principle in taxation appears in M ill (1848, Book V) and was axiomatically derived by $Y$ oung (1988).

With a few exceptions that will be indicated, all these rules satisfy the properties discussed below. We begin with some basic ones and devote the next section to properties concerning the concept of consistency.

\footnotetext{
${ }^{3}$ This equation has a unique solution when $E>0$. If $E=0$, any solution $\lambda$ is greater than or equal to the maximum claim and therefore $x_{i}=0$ for all $i$.
} 
A n allocation $x$ in $(E ; d)$ is said to be symmetric if whenever $d_{i}=d_{j}$, $x_{i}=x_{j}$.

A rule is symmetric if it always assigns symmetric allocations.

A rule $\phi$ is consistent if for any finite non-empty set $I$ of creditors,

$$
\begin{gathered}
\text { for all }(E ; d), d \in \mathbb{R}_{+}^{I}, \quad \text { for all } \varnothing \neq J \subseteq I, \\
\phi(E ; d)=x \Rightarrow x \mid J=\phi\left(\sum_{i \in J} x_{i}, d \mid J\right)
\end{gathered}
$$

where when $y \in \mathbb{R}_{+}^{I}, y \mid J$ is the projection of $y$ on $\mathbb{R}_{+}^{J}$.

A weaker condition is bilateral consistency, which requires (1) only for subsets $J$ containing exactly two creditors. The interpretation of consistency is as follows. Suppose that a rule $\phi$ assigns allocation $x$ to the bankruptcy problem $(E ; d)$. Suppose also that some subset of creditors wants to reallocate the total amount $\sum_{i \in J} x_{i}$ assigned to them. If we apply the same rule $\phi$ to allocate this amount among these creditors, each will get the amount originally assigned to him, provided $\phi$ is consistent. Consistency in the setup of bankruptcy problems was first discussed by A umann and M aschler (1985) and further analyzed by Y oung $(1987,1988)$. For an extensive survey, see Thomson (1996a).

A rule $\phi$ is monotone if for all bankruptcy problems $(E ; d)$ and $0 \leq E^{\prime} \leq E, \phi\left(E^{\prime} ; d\right) \leq \phi(E ; d)$.

Monotonicity says that a decrease in the estate does not benefit any creditor. A rule $\phi$ is strictly monotone if for all bankruptcy problems $(E ; d)$ and $0 \leq E^{\prime}<E$, if $d_{i}>0$ then $\phi_{i}\left(E^{\prime} ; d\right)<\phi_{i}(E ; d)$. Strict monotonicity says that a decrease in the estate leaves every non-zero creditor worse off. The rules in the above examples, with the exception of the proportional and equal sacrifice rules, do not satisfy strict monotonicity.

A rule $\phi$ is supermodular if for all $(E ; d)$ and $0 \leq E^{\prime} \leq E$, if $d_{i} \leq d_{j}$ then $\phi_{i}(E ; d)-\phi_{i}\left(E^{\prime} ; d\right) \leq \phi_{j}(E ; d)-\phi_{j}\left(E^{\prime} ; d\right)$. A supermodular rule allocates each additional dollar in an "order preserving" manner. ${ }^{4}$

A rule $\phi$ is anonymous if for every bankruptcy problem $\left(E,\left(d_{1}, \ldots, d_{n}\right)\right)$ and for all permutations $\pi$ of the set of creditors, we have

$$
\phi_{i}\left[E,\left(d_{\pi(1)}, \ldots, d_{\pi(n)}\right)\right]=\phi_{\pi(i)}\left[E,\left(d_{1}, \ldots, d_{n}\right)\right] \quad i=1,2, \ldots, n
$$

A nonymity requires that the rule $\phi$ be independent of the names of the creditors.

\footnotetext{
${ }^{4}$ E qual sacrifice rules relative to non-concave utility functions are not necessarily supermodular.
} 
The following lemmas will be useful in the rest of the paper.

Lemma 2.1. Any supermodular rule $\phi$ is symmetric.

Proof. Left to the reader.

LEMMA 2.2. Let $(E ; d)$ be a bankruptcy problem and let $i$ be a creditor with the highest claim. If $\phi$ is supermodular and $0 \leq E^{\prime}<E$, then $\phi_{i}(E ; d)>\phi_{i}\left(E^{\prime} ; d\right)$. That is, $i^{\prime} s$ award is strictly monotone in the estate.

Proof. Left to the reader.

\section{ON BILATERAL COMPARISONS, JUSTICE, AND CONSISTENCY}

Since every bankruptcy problem is a legal problem, its solutions should be guided by the principle of justice. Whatever form this principle may take, it should enable us to determine whether any one creditor received better or worse treatment than another at any given allocation. For example, if we believed, like A ristotle, that justice is proportionality, then we would say that $i$ is treated better than $j$ at allocation $x$ if $i$ receives a larger proportion of this claim than $j$ does. A ccording to this principle of justice, an allocation will treat $i$ and $j$ equally if they receive the same proportion of their claims. Obviously, we can think of other notions of justice, but in order to make these pairwise comparisons we clearly need only a bilateral principle.

A bilateral principle is a function that assigns a unique allocation to every two-person bankruptcy problem. We interpret this unique allocation as the just solution to the bilateral problem. We shall say that any other allocation in a two-person problem treats one creditor better than the other since it awards one creditor more than his "fair" share. A ny rule induces a bilateral principle, when it is projected on the class of 2-person bankruptcy problems. We denote a generic allocation rule by $\phi$ and its induced bilateral principle by $f$. M onotonicity, anonymity, and supermodularity of bilateral principles are defined in an obvious way.

$G$ iven a bankruptcy problem $(E ; d)$ and a bilateral principle $f$, we shall say that an allocation $x$ treats $i$ and $j$-equally if $\left(x_{i}, x_{j}\right)=f\left[x_{i}+x_{j}\right.$; $\left(d_{i}, d_{j}\right)$ ]. A n allocation in $(E ; d)$ is said to be $f$-just if it treats every two creditors $f$-equally. A umann and $M$ aschler (1985) showed that if a bilateral principle $f$ is monotone, then there is at most one $f$-just allocation for each bankruptcy problem. If a unique $f$-just allocation exists for any bankruptcy problem, then we can define the $f$-just rule to be the rule that assigns to each bankruptcy problem its unique $f$-just allocation. Conditions 
on a bilateral principle that guarantee the existence of the associated $f$-just rule can be found in Dagan and Volij (1996).

We explore some relations between $f$-justice and properties of allocation rules.

LEMMA 3.1. Let $f$ be a monotone bilateral principle and let $\phi$ be the f-just rule, then $\phi$ is consistent.

Proof. See A umann and M aschler (1985, Corollary 3.1).

LEMMA 3.2. Let $\phi$ be a monotone and bilateral consistent rule, and let $f$ be the bilateral principle induced by $\phi$. Then $\phi$ is the f-just rule.

Proof. Left to the reader.

COROLLARY 3.3. Let $f$ be a monotone bilateral principle. The f-just rule is the unique consistent rule that coincides with the bilateral principle $f$ in two-creditor problems. Moreover, when $\phi$ is monotone, consistency of $\phi$ is equivalent to bilateral consistency of $\phi$.

Proof. The first part follows directly from Lemma 3.2. As for the second part, if $\phi$ is monotone and bilaterally consistent, then by Lemma 3.2 it is the $f$-just rule, which by Lemma 3.1 is consistent.

The following lemma shows that monotonicity and supermodularity of the bilateral principle $f$ are properties inherited by the corresponding $f$-just rule.

LEMMA 3.4. Let $f$ be a bilateral principle and let $\phi$ be the f-just rule.

(a) If $f$ is supermodular, then $\phi$ is supermodular as well.

(b) If $f$ is monotone, then $\phi$ is monotone as well.

(c) If $f$ is supermodular, then $\phi$ is anonymous.

Proof. Let $(E ; d)$ be a bankruptcy problem and let $0 \leq E^{\prime} \leq E$. Let $x=\phi(E, d)$ and $x^{\prime}=\phi\left(E^{\prime}, d\right)$ be their corresponding $f$-just allocations.

(a) A ssume that $d_{i} \leq d_{j}$. We need to show that $x_{i}-x_{i}^{\prime} \leq x_{j}-x_{j}^{\prime}$. By definition of $\phi$ and by supermodularity of $f$ we have

$$
\begin{aligned}
x_{i}-x_{i}^{\prime} & =f_{i}\left(x_{i}+x_{j} ;\left(d_{i}, d_{j}\right)\right)-f_{i}\left(x_{i}^{\prime}+x_{j}^{\prime} ;\left(d_{i}, d_{j}\right)\right) \\
& \leq f_{j}\left(x_{i}+x_{j} ;\left(d_{i}, d_{j}\right)\right)-f_{j}\left(x_{i}^{\prime}+x_{j}^{\prime} ;\left(d_{i}, d_{j}\right)\right) \\
& =x_{j}-x_{j}^{\prime} .
\end{aligned}
$$

(b) A ssume by contradiction that for some creditor $i \in I$,

$$
x_{i}^{\prime}>x_{i}
$$


Since

$$
\sum_{k \in I} x_{k}^{\prime}<\sum_{k \in I} x_{k}
$$

there must exist a creditor $j$ with

$$
x_{j}>x_{j}^{\prime}
$$

Case 1. $x_{i}^{\prime}+x_{j}^{\prime} \leq x_{i}+x_{j}$. By definition of $\phi$ and monotonicity of $f$,

$$
x_{i}^{\prime}=f_{i}\left(x_{i}^{\prime}+x_{j}^{\prime} ;\left(d_{i}, d_{j}\right)\right) \leq f_{i}\left(x_{i}+x_{j} ;\left(d_{i}, d_{j}\right)\right)=x_{i}
$$

which is a contradiction to (2).

Case 2. $x_{i}^{\prime}+x_{j}^{\prime}>x_{i}+x_{j}$. By definition of $\phi$ and monotonicity of $f$

$$
x_{j}^{\prime}=f_{j}\left(x_{i}^{\prime}+x_{j}^{\prime} ;\left(d_{i}, d_{j}\right)\right) \geq f_{j}\left(x_{i}+x_{j} ;\left(d_{i}, d_{j}\right)\right)=x_{j}
$$

which contradicts (3).

(c) Let $\left\langle E ;\left(d_{1}, \ldots, d_{n}\right)\right\rangle$ be a bankruptcy problem and let $\pi$ : $\{1, \ldots, n\} \rightarrow\{1, \ldots, n\}$ be a permutation. Consider the auxiliary replica bankruptcy problem $\left\langle n E ;\left(d_{11}, \ldots, d_{1 n}, \ldots, d_{n 1}, \ldots, d_{n n}\right\rangle\right.$, where $d_{i k}=$ $d_{j k}=d_{k}$ for all $i, j, k \in\{1, \ldots, n\}$. L et $\left(x_{11}, \ldots, x_{1 n}, \ldots, x_{n 1}, \ldots, x_{n n}\right)$ be the corresponding allocation recommended by $\phi$. Since $f$ is supermodular, by part (a) and Lemma $2.1 \phi$ is symmetric. Therefore, $x_{i k}=x_{j k}=x_{k}$ for all $i, j, k \in\{1, \ldots, n\}$. Consequently, since $\pi$ is a permutation,

$$
\sum_{k=1}^{n} x_{1 k}=\sum_{k=1}^{n} x_{k \pi(k)}=E
$$

By Lemma 3.1, $\phi$ is consistent. Therefore, by the definition of $d_{i k}$ and the consistency of $\phi$,

$$
\begin{aligned}
\phi\left[E ;\left(d_{1}, \ldots, d_{n}\right)\right] & =\phi\left[\sum_{k=1}^{n} x_{1 k} ;\left(d_{11}, \ldots, d_{1 n}\right)\right] \\
& =\left(x_{11}, \ldots, x_{1 n}\right) \\
& =\left(x_{1}, \ldots, x_{n}\right) .
\end{aligned}
$$


A nalogously,

$$
\begin{aligned}
\phi\left[E ;\left(d_{\pi(1)}, \ldots, d_{\pi(n)}\right)\right] & =\phi\left[\sum_{k=1}^{n} x_{k \pi(k)} ;\left(d_{1 \pi(1)}, \ldots, d_{n \pi(n)}\right)\right] \\
& =\left(x_{1 \pi(1)}, \ldots, x_{n \pi(n)}\right) \\
& =\left(x_{\pi(1)}, \ldots, x_{\pi(n)}\right) .
\end{aligned}
$$

But since $\left\langle E ;\left(d_{1}, \ldots, d_{n}\right)\right\rangle$ is an arbitrary problem and $\pi$ is an arbitrary permutation, the above two equalities imply that $\phi$ is anonymous.

LemMA 3.5. Let $f$ be a monotone bilateral principle, let $(E ; d)$ be a bankruptcy problem, and let $x^{*}$ be its $f$-just allocation. Let $x$ be an allocation in $(E ; d)$ in which there are two creditors $i$ and $j$ with $x_{i} \leq x_{i}^{*}$ and $x_{j} \geq x_{j}^{*}$. Then, $f_{i}\left[x_{i}+x_{j} ;\left(d_{i}, d_{j}\right)\right] \geq x_{i}$. Moreover, if both inequalities are strict, then $f_{i}\left[x_{i}+x_{j} ;\left(d_{i}, d_{j}\right)\right]>x_{i}$.

Proof.

Case 1. $x_{i}+x_{j} \geq x_{i}^{*}+x_{j}^{*}$. By monotonicity and $f$-justice of $x^{*}$, $f_{i}\left[x_{i}+x_{j} ;\left(d_{i}, d_{j}\right)\right] \geq f_{i}\left[x_{i}^{*}+x_{j}^{*} ;\left(d_{i}, d_{j}\right)\right]=x_{i}^{*} \geq x_{i}$. H ence, $f_{i}\left[x_{i}+x_{j} ;\right.$ $\left.\left(d_{i}, d_{j}\right)\right] \geq x_{i}$.

Case 2. $x_{i}+x_{j}<x_{i}^{*}+x_{j}^{*}$. By monotonicity and $f$-justice of $x^{*}$, $f_{j}\left[x_{i}+x_{j} ;\left(d_{i}, d_{j}\right)\right] \leq f_{j}\left[x_{i}^{*}+x_{j}^{*} ;\left(d_{i}, d_{j}\right)\right]=x_{j}^{*} \leq x_{j}$. Hence, $f_{i}\left[x_{i}+x_{j} ;\right.$ $\left.\left(d_{i}, d_{j}\right)\right] \geq x_{i}$.

This proves the first part of the claim. As for the second part, it is proved analogously and is left to the reader.

Lemma 3.5 says that the $f$-just allocation of a bankruptcy problem is a good benchmark for bilateral comparisons: if at some allocation $x$ player $j$ gets more than the $f$-just allocation assigns to him and if player $i$ gets less than his $f$-just share, then $j$ must be receiving better treatment than $i$ at the allocation $x$. The next two lemmas have independent interest. They relate $f$-justice and the allocation prescribed by a consistent bankruptcy rule.

LEMMA 3.6. Let $(E ; d)$ be a bankruptcy problem, let $\phi$ be a consistent and monotone rule, and let $x$ be an allocation in $(E ; d)$. If there exists a creditor $i$ such that for all $j, x_{i}=\phi_{i}\left[x_{i}+x_{j} ;\left(d_{i}, d_{j}\right)\right]$, then $x_{i}=\phi_{i}(E ; d)$.

Proof. If $x_{i}>\phi_{i}(E ; d)$ then there exists a creditor $j$ with $x_{j}<\phi_{j}(E ; d)$. Hence, by Lemma $3.5, x_{i}>\phi_{i}\left[x_{i}+x_{j} ;\left(d_{i}, d_{j}\right)\right]$, contradicting the assumption of the lemma. A nalogous arguments are used if $x_{i}<\phi_{i}(E ; d)$.

LEMMA 3.7. Let $(E ; d)$ be a bankruptcy problem, let $\phi$ be a consistent, monotone, and supermodular rule and let $x$ be an allocation in $(E ; d)$. Let $i$ 
be a creditor with the highest claim. If for all creditors $j, x_{i}=\phi_{i}\left[x_{i}+x_{j}\right.$; $\left(d_{i}, d_{j}\right)$ ] then $x=\phi(E ; d)$.

Proof. By Lemma 3.6, $x_{i}=\phi_{i}(E ; d)$. Now assume there exists a creditor $j$ with $x_{j}<\phi_{j}(E ; d)$. By consistency, supermodularity, and Lemma 2.2, $x_{i}>\phi_{i}\left[x_{i}+x_{j} ;\left(d_{i}, d_{j}\right)\right]$, contradicting the assumption. So it must be that for all $j, x_{j} \geq \phi_{j}(E ; d)$. Since $x$ is an allocation, this implies that $x=$ $\phi(E ; d)$.

Lemma 3.7 says that if an allocation is such that all creditors are treated $f$-equally to one with a maximum claim, then this allocation is the $f$-just allocation. Thus, only $n$ equations (the $n-1$ conditions of $f$-equality with one of the highest claimants and the efficiency condition) are needed to calculate the $f$-just allocation of any $n$-creditor bankruptcy problem.

\section{A MULTILATERAL NONCOOPERATIVE MODEL}

Let $(E ; d)$ be a given bankruptcy problem. We are interested in defining an extensive form game for each bilateral principle $f$. The game, denoted by $G^{f}(E ; d)$ is defined as follows. Creditor 1 proposes an allocation $x$ in $\mathscr{A}(E ; d)$; following this proposal all the other creditors respond sequentially, either by accepting or rejecting the offer. The order of responses follow the protocol induced by the creditors' indices, namely creditor 2 is the first to respond and creditor $n$ is the last.

In order to define the players' payoffs, it is convenient to define the following state variable of "interim shares" for the proposer. Given a proposal $x$ and a profile of responses to it, define

$$
w_{1}=x_{1}
$$

and for $t=2, \ldots, n$,

$$
w_{t}= \begin{cases}w_{t-1} & \text { if } t \text { accepted } x \\ f_{1}\left[w_{t-1}+x_{t},\left(d_{1}, d_{t}\right)\right] & \text { if } t \text { rejected } x\end{cases}
$$

A n accepting creditor $t$ receives as a payoff $z_{t}=x_{t}$, a rejecting creditor $t$ receives $z_{t}=f_{t}\left(w_{t-1}+x_{t} ; d_{1}, d_{t}\right)$, and the proposer receives $z_{1}=w_{n}$.

$\mathrm{N}$ ote that creditor 1 's payoff is determined in several steps. The variable $w_{t}$ represents creditor 1 's interim share of the total estate after a proposal is made and the payoffs to all responders with indices no greater than $t$ are decided. That is, when creditor 1 proposes $x$ he determines $w_{1}$. To determine $w_{2}$, we need to know creditor 2's response to $x$. If creditor 2 accepts it, $w_{2}=w_{1}$ (creditor 2 finds no grounds to appeal to the bilateral 
court about the unfairness of the proposal); otherwise, $w_{2}=f_{1}\left[w_{1}+\right.$ $x_{2} ;\left(d_{1}, d_{2}\right)$ ] because creditor 2 "renegotiates" with creditor 1 over his fair share of $w_{1}+x_{2}$, and so on.

In sum, creditor 1 may be interpreted as the administrator of the estate. He proposes an allocation $x$ and he is entitled to award the proposed shares to the accepting creditors. However, he must be open to counterarguments made by those who reject the offer, who may hold him responsible for his proposal before the court (by claiming their fair share of the amount $\left.w_{t-1}+x_{t}\right)$. That is, we assume that the bilateral principle $f$ is commonly accepted in society, or that resorting to litigation is an outside option for the creditors. The administrator then receives the remainder of the estate after all the other creditors received their shares.

Note that every play of the game $G^{f}(E, d)$ results in an allocation of $(E, d)$. To see this let $\sigma$ be an arbitrary strategy profile, let $\left(x_{1}, x_{2}, \ldots, x_{n}\right)$ be the proposal, let $\left\{w_{t}\right\}_{t=1}^{n}$ be the associated interim payoff sequence, and let $\left(z_{1}, z_{2}, \ldots, z_{n}\right)$ be the corresponding payoff vector. By the definition of the payoff vector, $0 \leq z_{t} \leq d_{t}$, for $t=1,2, \ldots, n$. Therefore it is enough to show that $\sum_{t=1}^{n} z_{t}=E$. N ote that by the definition of $w_{t}$, for $t=1,2, \ldots, n$ and of the payoff vector,

$$
z_{t}=w_{t-1}-w_{t}+x_{t} \quad \text { for } t=2,3, \ldots, n .
$$

Therefore,

$$
\begin{aligned}
\sum_{t=1}^{n} z_{t} & =\sum_{t=2}^{n} z_{t}+w_{n} \\
& =\sum_{t=2}^{n}\left(w_{t-1}-w_{t}+x_{t}\right)+w_{n} \\
& =w_{1}+\sum_{t=2}^{n} x_{t} \\
& =\sum_{t=1}^{n} x_{t} \\
& =E .
\end{aligned}
$$

The introduction of the bilateral principle to calculate the proposer's payoff may seem arbitrary. However, we want to emphasize that our purpose is not to characterize a certain consistent rule or any bilateral principle; instead, we are more interested in the relationship between the bilateral principles and their consistent generalizations. For a model in which the bilateral principle does not appear in the extensive form game see Serrano (1995), who characterized the contested garment consistent rule.

N ow we are ready to state the main result of this paper. 
THEOREM 4.1. Let $(E ; d)$ be a bankruptcy problem where creditor 1 has the highest claim, let $f$ be a monotone and supermodular bilateral principle, and let $\phi$ be the f-just rule. The unique Nash equilibrium outcome of $G^{f}(E ; d)$ is $\phi(E ; d)$. Moreover, it can be supported by a pure strategy subgame perfect equilibrium.

Some remarks are in order.

(1) The theorem holds for the case in which the proposer is a creditor with a highest claim. Otherwise uniqueness is not obtained (see Example 6.1 in Section 6). On the other hand, as will be shown in Section 6, for strictly monotone rules the identity of the proposer is of no importance. $\mathrm{N}$ ote also that the result is invariant to the order of responders.

(2) The result does not use any refinement of $\mathrm{N}$ ash equilibrium. This is in contrast to other models that relate noncooperative models with pure bargaining problems. When the underlying model is more complex, as in general cooperative games, a refinement of subgame perfect equilibrium is usually needed in order to get uniqueness (see, for example, $\mathrm{H}$ art and M as-Colell (1996) and Gul (1989)).

(3) Unlike some other models, which provide a noncooperative view of a cooperative solution concept, our result yields a noncooperative view of a large family of allocation rules for bankruptcy problems. The two critical properties that characterize this family are consistency and monotonicity. These two properties guarantee that the allocation assigned by the consistent rule can be supported by a $\mathrm{N}$ ash equilibrium. These properties are the ones that drive the results of other consistency-based non-cooperative mechanisms (see, for example, Krishna and Serrano (1996) and Chae and $Y$ ang (1994)).

(4) O ur result holds for the whole family of bankruptcy problems. This is in contrast to other models, such as those mentioned in the previous remark, where the unique subgame-perfect equilibrium outcomes converge to the Nash bargaining solution agreement for "dividing a dollar" bargaining problems, in which the Nash solution is monotone (see Chun and Thomson, 1988). The reason why these models do not yield a similar result in all bargaining problems becomes apparent: the $\mathrm{N}$ ash bargaining solution is not monotone in general. ${ }^{5}$

(5) The unique equilibrium agreement is not achieved necessarily after unanimous agreement. This feature of the model agrees with the

\footnotetext{
${ }^{5}$ W hen the Nash solution is not monotone, the strategies proposed by K rishna and Serrano (1996) and by Chae and $Y$ ang (1994) do not constitute even a N ash equilibrium. The proposer could find a profitable deviation by offering more than his equilibrium share to one of the responders, in the hope of benefiting from a bigger share in a smaller pie.
} 
consistency principle. As we know, after applying a consistent rule any subset of agents is indifferent between accepting their shares and renegotiating among themselves.

(6) The possible emergence of an equilibrium without unanimous agreement is due to the fact that there is no cost of renegotiation, such as discounting, fixed renegotiation fees, or the random elimination of players.

Proof of Theorem 4.1. We denote $\phi(E ; d)$ by $x^{*}$ and equilibrium outcomes by $z$. The proof follows from the following steps.

STEP 1. In any equilibrium of $G^{f}(E ; d), z_{1} \geq x_{1}^{*}$.

Proof. Creditor 1 can guarantee a payoff of $x_{1}^{*}$ simply by proposing $x^{*}$. To see this, note that if the proposal is $x^{*}$, then $w_{1}=x_{1}^{*}$, and if $w_{t-1}=x_{1}^{*}$ then by the definition of $w_{t}$ and consistency of $\phi, w_{t}=x_{1}^{*}$ independent of the responses. So in any equilibrium, the proposer gets at least $x_{1}^{*}$.

StEP 2. Let $z$ be an equilibrium outcome of $G^{f}(E ; d)$. Then $f_{1}\left(z_{1}+z_{t}\right.$; $\left.\left(d_{1}, d_{t}\right)\right) \geq z_{1}$ for all $t>1$.

Proof. Let $\sigma$ be an equilibrium of $G^{f}(E ; d)$ and let $\left\{w_{t}\right\}_{t=1}^{n}$ be the interim payoff sequence determined by the equilibrium path. Given the equilibrium proposal $x$ and the responses of the others, creditor $t$ 's payoff is either $f_{t}\left(w_{t-1}+x_{t} ;\left(d_{1}, d_{t}\right)\right)$ or $x_{t}$ depending on whether he rejects or accepts the proposal, respectively. Therefore in equilibrium we must have

$$
z_{t}=\max \left\{f_{t}\left(w_{t-1}+x_{t} ;\left(d_{1}, d_{t}\right)\right), x_{t}\right\}
$$

and

$$
w_{t}=w_{t-1}+x_{t}-z_{t} .
$$

By (6) and the definition of $z_{1}$ we have

$$
z_{1}=w_{n} \leq w_{t} \quad \text { for all } t .
$$

Given the identity $f_{1}\left(z_{1}+z_{t} ;\left(d_{1}, d_{t}\right)\right)+f_{t}\left(z_{1}+z_{t} ;\left(d_{1}, d_{t}\right)\right)=z_{1}+z_{t}$, it follows from (7) and monotonicity that

$$
f_{1}\left(z_{1}+z_{t} ;\left(d_{1}, d_{t}\right)\right)+f_{t}\left(w_{t}+z_{t} ;\left(d_{1}, d_{t}\right)\right) \geq z_{1}+z_{t} .
$$

By (6), $f_{1}\left(z_{1}+z_{t} ;\left(d_{1}, d_{t}\right)\right)+f_{t}\left(w_{t-1}+x_{t} ;\left(d_{1}, d_{t}\right)\right) \geq z_{1}+z_{t}$ and by (5), $f_{1}\left(z_{1}+z_{t} ;\left(d_{1}, d_{t}\right)\right) \geq z_{1}$.

Step 3. $z_{1} \leq x_{1}^{*}$.

Proof. If $z_{1}>x_{1}^{*}$, since $z$ and $x^{*}$ are both allocations, there must be some creditor $t$ with $z_{t}<x_{t}^{*}$. Then, by Lemma 3.5 we must have $f_{1}\left(z_{1}+z_{t} ;\left(d_{1}, d_{t}\right)\right)<z_{1}$, contradicting Step 2 . 
StEP 4. $z=x^{*}$.

Proof. A ssume by contradiction that $z \neq x^{*}$. Then there is a creditor $t$ with $z_{t}<x_{t}^{*}$. By Steps 1 and 3 this creditor cannot be the proposer. Hence, by Steps 2, 3, and 1 and by supermodularity of $f$ (L emma 2.2) we have that $f_{1}\left(x_{1}^{*}+x_{t}^{*} ;\left(d_{1}, d_{t}\right)\right)>f_{1}\left(z_{1}+z_{t} ;\left(d_{1}, d_{t}\right)\right) \geq z_{1}=x_{1}^{*}$. This is a contradiction to the $f$-justice of $x^{*}$.

As for existence, it can easily be seen that the following strategy profile constitutes a subgame perfect equilibrium:

(I) Creditor 1 proposes $x^{*}$; and

(II) Creditor $t$ accepts a proposal $x$ if and only if $x_{t} \geq f_{t}\left[w_{t-1}+x_{t}\right.$; $\left(d_{1}, d_{t}\right)$ ], where $w_{t-1}$ is defined in 4 .

This concludes the proof of the theorem.

\section{COALITIONAL STABILITY OF THE EQUILIBRIA}

In the game presented in Section 4 creditors may exit with the share proposed to them simply by accepting the proposal. The question arises of whether the equilibria are coalitionally stable. Could the proposer offer a larger share of the pie to a responder in the hope of profiting from a joint deviation? To answer this question we consider the game $\Gamma^{f}(E ; d)$ in strategic form that corresponds to the game $G^{f}(E ; d)$ in extensive form.

We first consider any kind of coalitional deviation and ask if the subgame perfect equilibria of our model are strong $N$ ash in $\Gamma^{f}(E ; d)$ (A umann, 1959). This requires that no coalition of players have a joint deviation which leaves all its members better off. We find that the equilibria of our model fail in general to be strong. This is illustrated in the following example.

EXAMPLE 5.1. Consider the bankruptcy problem problem $(E ; d)=$ $\langle 99 ;(100,100,100)\rangle$. Since this problem is symmetric, for all symmetric bilateral principles the game $G^{f}(E ; d)$ is the same. Clearly, the $f$-just allocation in this problem, and hence the unique subgame perfect equilibrium outcome, is $(33,33,33)$. Consider the following deviation by the first and third creditors: The proposer offers $x=(0,1,98)$, and the third creditor, who was offered 98 dollars, rejects it. This deviation yields more than 33 both to creditor 1 and creditor 3, no matter how creditor 2 responds. Therefore no equilibrium of $G^{f}(E, d)$ is a strong equilibrium.

Note that although this deviation improves creditor 3's payoff relative to the $f$-just allocation, he can do even better by deviating from his joint deviation with creditor 1 and accepting 1's offer. This makes the above 
deviation unstable. Examples of this sort motivated the alternative concept of coalitional stability known as coalition-proof $\mathrm{N}$ ash equilibrium, introduced by Bernheim et al. (1987).

The following definition ${ }^{6}$ refers to games in normal form, $\Gamma=$ $\left(I,\left(S_{i}\right)_{i \in I},\left(g^{i}\right)_{i \in I}\right)$, where $I$ is the set of players, $S_{i}$ is the strategy set of player $i$, and $g^{i}$ is the payoff function of player $i$. L et $J$ be a coalition, that is, $\varnothing \neq J \subseteq I$. We denote $S_{J}=\times_{i \in J} S_{i}$. A lso, if $\sigma \in S:=\times_{i \in I} S_{i}$ is a list of strategies, $\sigma_{J}$ denotes the restriction of $\sigma$ to coalition $J$. Given a game $G$, a list $\sigma$ of strategies (one for each player), and a coalition $J$ of players, an internally consistent improvement of $J$ upon $\sigma$ is defined by induction on $|J|$. If $J=\{i\}$ for some $i$ in $I$, then $\tau_{i} \in S_{i}$ is an internally consistent improvement of $J$ upon $\sigma$ if $g^{i}\left(\tau_{i}, \sigma_{I \backslash\{i\}}\right)>g^{i}(\sigma)$. If $|J|>1$ then $\tau_{J} \in S_{J}$ is an internally consistent improvement upon $\sigma$ if (i) $g^{i}\left(\tau_{J}, \sigma_{I \backslash J}\right)>g^{i}(\sigma)$ for all $i$ in $J$, and (ii) no $T \subset J, T \neq \varnothing$, has an internally consistent improvement upon $\left(t_{J}, \sigma_{I \backslash J}\right)$. The profile $\sigma$ is a coalition-proof Nash equilibrium if no $J \subseteq I, J \neq \varnothing$, has an internally consistent improvement upon $\sigma$.

In contrast to strong $\mathrm{Nash}$, the coalition-proof $\mathrm{Nash}$ equilibrium requires that no coalition should have a profitable and self-enforcing deviation.

THEOREM 5.2. Let $f$ be a monotone and supermodular bilateral principle and let $\phi$ be the f-just rule. For all bankruptcy problems $(E ; d)$, all the subgame-perfect equilibria of $G^{f}(E ; d)$ are coalition-proof equilibria of $\Gamma^{f}(E ; d)$.

Proof. Let $(E ; d)$ be a bankruptcy problem and let $f$ be a monotone and supermodular bilateral principle. Let $\sigma$ be a subgame perfect equilibrium and suppose there is a coalition of creditors that has an internally consistent improvement upon $\sigma$.

A ssume that the proposer does not belong to the deviating coalition. Let $k$ be the member of the deviating coalition who has the lowest index, that is, creditor $k$ is the first to respond among the deviators. Consider the node of the deviation path in which creditor $k$ has to respond. Since his payoff is independent of the strategies of the creditors with higher indices than $k$, the mere existence of the assumed improvement contradicts the fact that $\sigma$ is a $\mathrm{N}$ ash equilibrium. Therefore, the proposer must belong to the deviating coalition.

Thus, suppose that the proposer is a member of the deviating coalition and let $x$ be the proposal made in the deviation. Denote by $\left\{v_{t}\right\}$ the sequence of interim payoffs of the proposer determined by the joint

\footnotetext{
${ }^{6}$ It is taken from Peleg and Tijs (1996).
} 
deviation and by $\left\{w_{t}\right\}$ the sequence corresponding to the case in which all responders follow their equilibrium strategies after $x$ has been proposed.

We claim that $v_{t}=w_{t}$ for all $t$. The proof is by induction. By definition $v_{1}=w_{1}=x_{1}$. Assume now that the equality is true for all $t<s$ and consider the node where creditor $s$ has to respond. If creditor $s$ does not belong to the coalition of deviators, then it is clear that $w_{s}=v_{s}$ because $v_{s-1}=w_{s-1}$ and he does not deviate. If creditor $s$ belongs to the set of deviators, then since the deviation is internally consistent it means that, in particular, creditor $s$ cannot find it profitable to deviate from the joint deviation. Therefore $v_{s} \geq w_{s}$. On the other hand, since $\sigma$ is a subgame perfect equilibrium and $v_{s-1}=w_{s-1}$, it must be the case that $v_{s} \leq w_{s}$. Hence $w_{s}=v_{s}$.

This means that, following the proposal $x$, and whether the deviating responders follow the deviation or not, the proposer's payoff is the same. But then, since $\sigma$ is a subgame perfect equilibrium, by Theorem 1 $v_{n}=w_{n} \leq x_{1}^{*}$ which contradicts the fact that the proposer belongs to a coalition that has an internally consistent improvement upon $\sigma$.

Remark. In fact, the proof above also shows that all the subgame-perfect equilibria of $G^{f}(E ; d)$ are perfectly coalition-proof equilibria of the same game. (For a definition of perfectly coalition-proof equilibrium, see Bernheim et al. (1987)). This result, however, is not surprising since Peleg (1992) showed that for extensive form games with perfect information, the set of subgame perfect equilibria coincides with the set of perfectly coalition-proof equilibria.

\section{STRICTLY MONOTONE RULES}

The uniqueness result in Theorem 4.1 is driven by the strict estate monotonicity of rule $\phi$ with respect to the highest claim (L emma 2.2). This is why it is important that the proposer should be a creditor with the highest claim. If the proposer's component $\phi_{1}$ was not strictly monotone in the estate, multiplicity of subgame-perfect equilibrium outcomes might arise, as shown by the following example:

EXAMPLE 6.1. Let $(E ; d)=\langle 100 ;(10,100,100)\rangle$. If $\phi$ is the constrained equal award rule, $\phi(E ; d)=(10,45,45)$. The reader can check that all the outcomes of the form $(10,45-a, 45+a)$ for $-35 \leq a \leq 35$ can be supported by subgame perfect equilibria of the corresponding extensive form game.

If we confine ourselves to consistent and strictly monotone rules, the main result can be generalized to the case in which the proposer is any creditor with a positive claim. These results are stated formally in Theorem 6.2 . 
THEOREM 6.2. Let $(E ; d)$ be a bankruptcy problem, let $f$ be a strictly monotone bilateral principle, and let $\phi$ be the f-just rule. Suppose creditor 1 has a positive claim. The unique subgame perfect equilibrium outcome of $G^{f}(E ; d)$ is $\phi(E ; d)$. Moreover, all subgame-perfect equilibria of $G^{f}(E ; d)$ are coalition-proof equilibria of $\Gamma^{f}(E ; d)$.

Proof. The proof is identical to the Proofs of Theorems 4.1 and 5.2 with the only exception that in Step 4 of Theorem 4.1, the words "supermodularity (L emma 2.2)" should be replaced by "strict monotonicity."

\section{CONCLUDING REMARKS}

By giving a noncooperative view of a wide class of bankruptcy rules, we believe we have provided additional support to the idea that the property of consistency is useful in the Nash Program for cooperative games. On the other hand, consistency alone, without the assistance of monotonicity, is insufficient to reach the results. Thus, construction of consistency based noncooperative models that support consistent cooperative solution concepts which are not monotone seems to us a difficult task. Therefore there might be problems in supporting the nucleolus or the Nash bargaining solution on general pies by means of a noncooperative model. ${ }^{7}$ In the bankruptcy model, however, monotonicity is a natural requirement. M oreover, it is almost implied by consistency: Y oung (1987, Lemma 1) showed that if a rule is symmetric, continuous, and consistent, then it is also monotone.

\section{REFERENCES}

A umann, R. J. (1959). "A cceptable Points in General Cooperative $n$-Person Games," in Contributions to the Theory of Games, Vol. 4 (A. W. Tucker and R. D. Luce, Eds.), pp. 287-324, Princeton, NJ : Princeton U niversity Press.

A umann, R. J ., and M aschler, M . (1985). "G ame Theoretic A nalysis of a Bankruptcy Problem from the Talmud," J. Econom. Theory 36, 195-213.

Bernheim, B. D., Peleg, B., and Whinston, M. D. (1987). "Coalition-Proof Nash Equilibria I Concepts," J. Econ. Theory 43, 1-12.

Chae, S., and Y ang, J.-A . (1994). "An N-Person Pure Bargaining Game," J. Econ. Theory 62, 86-102.

Chun, Y ., and Thomson, W. (1988). "M onotonicity Properties of Bargaining Solutions when A pplied to Economics," Math. Soc. Sci. 15, 11-27.

${ }^{7} \mathrm{H}$ art and M as-Colell (1996) support the Nash bargaining solution for general pies via a noncooperative model, but their model is not "consistency based." 
Dagan, N., and Volij, O. (1996). "Bilateral Comparisons and Consistent Fair Division Rules in the Context of Bankruptcy Problems," Int. J. Game Theory, forthcoming.

D agan, N ., Serrano, R ., and V olij, O . (1995). "Feasible Implementation of Taxation M ethods," W orking Paper 95-14, D ept. of E conomics, Brown U niversity.

Gul, F. (1989). "Bargaining F oundations of the Shapley V alue," Econometrica, 57, 81-95.

H art S., M as-Colell, A . (1996). "Bargaining and V alue," Econometrica 64, 357-380.

K rishna, V ., and Serrano, R. (1996). "M ultilateral Bargaining," Rev. Econ. Studies 63, 61-80.

Lensberg, T. (1988). "Stability and the Nash Solution," J. Econ. Theory 45, 330-341.

M ill, J. S. (1848). Principles of Political Economy. N ew Y ork: Collier and Sons 1900), rev. ed.

Peleg, B. (1986). "On the R educed Game Property and Its Converse," Int. J. Game Theory 15, 187-200.

Peleg, B. (1992). "On Perfectly Coalition-Proof Nash Equilibria," in Equilibrium and Dynamics (M. M ajumdar, Ed.). New Y ork: St. M artin's Press.

Peleg, B., and Tijs, S. (1996). "The Consistency Principle for Games in Strategic Form," Int. J. Game Theory, 25, 13-34.

Pineles, H. M . (1861). Darkah Shel Torah. V ienna: Forster [in H ebrew].

Serrano, R. (1995). "Strategic Bargaining, Surplus Sharing Problems and the Nucleolus," J. Math. Econ. 24, 319-329.

Thomson, W. (1990). "The Consistency Principle," in Game Theory and Applications (T. Ichiishi, A. Neyman, and Y. Tauman, Eds.). San Diego: A cademic Press.

Thomson, W. (1996a). "A xiomatic A nalyses of Bankruptcy and Taxation Problems: A Survey," Math. Social Sci., forthcoming.

Thomson, W. (1996b). "Consistent Allocation R ules," Working Paper 418. R ochester Center for E conomic R esearch.

Y oung, H. P. (1987). "On Dividing an A mount A ccording to Individual Claims or Liabilities," Math. Oper. Res. 12, 398-414.

Y oung, H. P. (1988). "D istributive J ustice in Taxation," J. Econom. Theory, 44, 321-335. 